\title{
Effect of the Partner Lounge Initiative on male partner involvement in HIV/AIDS services uptake among HIV-positive pregnant women in Cross River State, Nigeria: A pilot study
}

\author{
Philip Imohi ${ }^{1}$, Frank Eyam ${ }^{1}$, Antor O. Ndep ${ }^{2}$, Kingsley Obase ${ }^{1}$, Cajetan Obi ${ }^{1}$, Peter Agada ${ }^{1}$, \\ Nnennaya Igwe ${ }^{1}$, Augustine Idemudia ${ }^{1}$, Atim Udo ${ }^{1}$, Joshua Nwankon Pam ${ }^{1}$, Ogbu Ngim ${ }^{1}$ \\ ${ }^{1}$ Department of HIV/AIDS Research \\ Enareh Public Health Consultancy \\ Calabar, Cross River State, Nigeria \\ ${ }^{2}$ Department of Public Health, \\ Faculty of Allied Medical Sciences, \\ University of Calabar, Calabar, Nigeria
}

\begin{abstract}
Corresponding Author: Dr. Antor O. Ndep. Department of Public Health, University of Calabar, Calabar, Nigeria. Email: drndep@gmail.com Phone: +234-906-0381917
\end{abstract}

DOI: $10.31364 / \mathrm{SCIRJ} / \mathrm{v} 8 . \mathrm{i} 4.2020 . \mathrm{P} 0420760$

http://dx.doi.org/10.31364/SCIRJ/v8.i4.2020.P0420760

\begin{abstract}
Background: One in four children born with HIV infection globally, is born in Nigeria! Poor Ante-natal clinic (ANC) attendance has been implicated. Objective: To examine the significance of male involvement in ANC service utilization and ART adherence via the Partners' Lounge Initiative (PLI). Methods: Male partners were treated to a Partner Lounge Intervention (PLI); a comfortable, well-furnished waiting area while the women accessed ANC/ART services. Results: Pre-PLI, half of the respondents registered for antenatal care during their second trimester. Only 16.7\% never missed ANC/ART clinic appointments while $41.7 \%$ missed once, $\mathbf{4 1 . 7 \%}$ of the males never accompanied their partners to clinic, 50\% accompanied theirs only once. At post-PLI, 53.3\% enrolled for ANC during the second trimester, $60 \%$ and $66.7 \%$ never missed ANC and ART clinics respectively, none of the women came to clinic unaccompanied by their male partners; $26.7 \%$, were accompanied once, $40 \%$, twice and $33.3 \%$, three or more times. Sero-discordance was identified in both groups; $25 \%$ of males at pre-PLI and $53.3 \%$ at post-PLI were unreactive. Pre- and post-PLI difference in missed ART appointments was significant $(p=0.017)$. Conclusion/Recommendations: PLI increased male involvement in their partners' ANC/ART clinic visits. The role of sero-discordance in ART adherence may require further inquiry.
\end{abstract}

Keywords: Male Involvement, ANC, ART, PMTCT, Partners'Lounge Initiative, sero-discordance

\section{INTRODUCTION}

Globally, strategies have been implemented to reduce the burden of HIV/AIDS on affected communities. Among those living with HIV/AIDS, majority are young people between the age of 15 -34 [1]. Meaning that a large majority of the working force are affected. Reducing new HIV infection by getting people tested and linked to care has proved to be an effective method of reducing the burden of HIV on individuals, their families, the communities they live and work in and ultimately the countries' economies. Some of these strategies applied since the $80 \mathrm{~s}$ to early 2000s have been mainly successful in reducing the new cases. This trend of reducing new cases has stalled in recent years in Europe and America but not in Sub-Saharan Africa. Nigeria's contribution to the global burden of HIV is a whopping 1.9 million persons! High risk groups include commercial sex workers, injection drug users and inter-state commercial drivers. While these groups are highly visible and targeted interventions have recorded minimal successes, it is apparent that other high-risk groups who may be engaged in risky sexual behaviours are yet to be identified. The population of people living with HIV/AIDS is becoming younger with the 15-34 age bracket having the highest prevalence! One in every four children born with HIV in the world, is born in Nigeria [2]. Poor Ante-natal clinic (ANC) attendance has been implicated in numerous poor maternal and child indices. In Nigeria just as in the rest of Sub-Saharan Africa, research has shown that that male involvement (MI) in the care of their pregnant partners yields positive outcomes for both maternal and child indices yet MI is still very low [3]. 
HIV positive women are at a high risk of infecting their unborn child during pregnancy. Prevention-of-Mother-To-ChildTransmission (PMTCT) of HIV services have been successful in reducing neonatal HIV infection reducing neonatal morbidity and mortality due to HIV. The main objectives of PMTCT program are to reduce in-utero transmission of the virus to the unborn child, promote access to anti-retroviral therapy (ART) for the HIV positive pregnant woman regardless of viral load or stage of infection, promote access to care for HIV positive infants, preserve the family unit and reduce the number of orphans due to HIV/AIDS [4]. ANC attendance is closely linked with PMTCT, since the directly observed therapy (DOTS) is the method used in administering ART in the PMTCT intervention. Studies have shown that women whose male partners accompanied them to ANC tended [5] to consistently take their ARVs can achieve viral load suppression enough to reach zero levels of sexually transmitting HIV [6]. Men could be critical partners in reversing the trend of poor utilization of ANC services in health facilities and by extension help increase uptake of PMTCT. However, getting male partners involved in what is traditionally viewed as a woman's responsibility may take innovative approaches [5].

\section{OBJECTIVE}

This study examined the effect of the Partner Lounge Initiative (PLI) on male partner involvement in ANC service utilization and ART adherence by HIV positive pregnant women.

\section{METHODS}

For the pre-intervention group, pregnant women regardless of the gestational age, were approached to be part of this study during their ANC enrolment at a secondary health facility in Calabar. In Cross River State, all pregnant women receive HIV counselling and testing services. Inclusion criteria for this study was, a pregnant woman whose HIV test is positive and she agrees to be part of the study and to bring her male partner during subsequent ANC visits. Recruitment took place from June to December 2017. Twelve women met the inclusion criteria for the pre-intervention group and were followed up until they gave birth.

In March 2018, a comfortable and well-furnished waiting area for male partners of pregnant women (Partner Lounge) was set up within the antenatal/family planning clinic section of a secondary health facility leading to the birth of the Partner Lounge Initiative (PLI). HIV positive pregnant woman who registered for ANC, between March and December 2018 who agreed to participate and to bring their male partners in subsequent ANC were recruited to participate in the study and were followed up until they gave birth. Fifteen women met the inclusion criteria for the post-intervention group. Accompanying male partners of the 16 pregnant women accessing ANC services were treated to a friendly ambience, where they watched sports or news on television and had access to newspapers and other magazines. Male partner attendance figures were assessed at both the ANC and ART clinics.

\section{RESULTS}

The mean age \pm standard deviation (SD) of the women in the pre-PLI group was $29.3 \pm 5.2$ years, the youngest was 22 years and the oldest was 35 years. Majority, 58.3\% were in the 30-35 age group, 66.7\% had a secondary school education and the earliest gestational age at the time of ANC enrolment was 12 weeks while the oldest was 32 weeks. Fifty percent of the respondents registered for antenatal care during their second trimester. The minimum number of scheduled ANC/ART clinic visits was four and the maximum was nine. All 12 participants accepted their HIV results and agreed to enrol in care. For the male partners at pre-PLI, the mean age \pm SD was $39.1 \pm 4.6$, the youngest being 31 years old. Only $16.7 \%$ never missed both ANC and ART clinic appointments while $41.7 \%$ missed both clinic appointments only once. For the pre-PLI group, $41.7 \%$ of the males never accompanied their pregnant partners to clinic while 50\% accompanied their pregnant partners only once.

For the post-PLI group, the mean age \pm SD of the pregnant women was $29.8 \pm 6.7$, the youngest was 16 years while the oldest was 43 years old. Forty percent were within the 30-35 age group and $73.3 \%$ had a secondary education. The earliest gestational age at the time of ANC enrolment was 12 weeks while the oldest was 32 weeks and 53.3\% enrolled for ANC during the second trimester. Thirteen of the fifteen eligible respondents accepted their HIV-positive test results and were enrolled in care on the dame day. Two were in denial and subject to follow up counselling. As with the pre-PLI group, the minimum number of scheduled ANC/ART clinic visits was four and the maximum was nine. Sixty percent and $66.7 \%$ never missed any clinic appointments for ANC and ART respectively. For the male partners at post-PLI, the mean age \pm SD was $38.5 \pm 4.7$ [See Table 1]. For male involvement, $26.7 \%$ of the men accompanied their pregnant partners once, $40 \%$, twice and $33.3 \%$ accompanied their partners three or more times [see Figure 1]. All male partners were counselled and tested and the HIV test result revealed that $25 \%$ of males at pre-PLI and $53.3 \%$ at post-PLI were unreactive. A comparison of pre- and post-PLI pre- and post-PLI number of missed ART appointments was significant $(\mathrm{p}=0.017)$.

\section{DISCUSSION}


Since the advent of HIV/AIDS, researchers have engaged in seeking the best possible interventions that could help curb the spread of the epidemic. One of such interventions is PMTCT. In a meta-analysis done in 2017, researchers found that male involvement is critical in the uptake of PMTCT however, the methods used to achieve male involvement varied [7,8]. Invitation letters and verbal encouragement of the male partners seemed to work best in attracting them to accompany their pregnant partners to ANC and ART clinics [7]. Early ANC enrolment is another important factor that could turn the tide on the population of children born with HIV. In this study, PLI led to increase in male involvement in their partners' ANC visits which corroborates some researchers' findings that male involvement in the care of their HIV positive pregnant partners led to desirable pregnancy outcomes $[4,9]$ which could increase uptake of PMTCT over time. Getting pregnant women to attend ANC is still a major challenge. A UNICEF report showed only 49.1\% of women aged 15-49 attended ANC four or more times (WHO standard) while $65.8 \%$ attended ANC at least once during their last pregnancy that led to a live birth [4, 10]. Desirable health outcomes for HIV positive pregnant women and their unborn children is of the utmost importance however, using the PLI means that the participating women have disclosed their HIV status to their partners. Studies have shown that disclosure status affects uptake of HIV/AIDS services [11, 12] which includes ANC attendance and subsequent uptake of PMTCT.

While this study is limited to just one facility and uses simple frequencies of attendance to measure MI, a more current measurement scale [5] for MI may shed more light on the current status of this important factor and determinant of PMTCT. In addition, a comparative study between rural and urban health care facilities' implementation of PLI could shed more on MI and its relationship with ANC and PMTCT uptake among HIV positive pregnant women in Cross River State, Nigeria. Serodiscordance seem to be a major characteristic among the couples in this study at pre- and post-PLI. How this characteristic affect ART adherence as well as uptake of PMTCT and other HIV/AIDS services may require further inquiry.

Table 1: Respondents' characteristics

\begin{tabular}{|c|c|c|}
\hline \multirow[t]{2}{*}{ Variable } & \multicolumn{2}{|c|}{ Frequency $\mathrm{n}(\%)$} \\
\hline & $\begin{array}{l}\text { Pre-Partner } \\
\text { Lounge Initiative } \\
n=12\end{array}$ & $\begin{array}{l}\text { Post-Partner } \\
\text { Lounge } \\
\text { Initiative } \\
\mathrm{n}=15 \\
\end{array}$ \\
\hline \multicolumn{3}{|l|}{ Age group } \\
\hline $15-19$ & $0(0)$ & $1(6.7)$ \\
\hline $20-25$ & $3(25.0)$ & $3(20.0)$ \\
\hline $26-29$ & $2(16.7)$ & $3(20.0)$ \\
\hline $30-35$ & $7(58.3)$ & $6(40.0)$ \\
\hline $36-39$ & $0(0)$ & $1(6.7)$ \\
\hline 40 and older & $0(0)$ & $1(6.7)$ \\
\hline \multicolumn{3}{|l|}{ Educational qualification } \\
\hline No formal education & $0(0)$ & $1(6.7)$ \\
\hline Secondary & $8(66.7)$ & 11(73.3) \\
\hline OND/NCE & $1(8.3)$ & $1(6.7)$ \\
\hline $\mathrm{HND} / \mathrm{BSc}$ & $3(25.0)$ & $2(13.3)$ \\
\hline \multicolumn{3}{|l|}{$\begin{array}{l}\text { Gestational age at ANC } \\
\text { enrolment }\end{array}$} \\
\hline First Trimester & $3(25.0)$ & $2(13.3)$ \\
\hline Second Trimester & $6(50.0)$ & $8(53.3)$ \\
\hline Third Trimester & $3(25.0)$ & $5(33.3)$ \\
\hline \multicolumn{3}{|l|}{$\begin{array}{l}\text { Number of times missed ANC } \\
\text { appointment }\end{array}$} \\
\hline Never & $2(16.7)$ & $9(60.0)$ \\
\hline Once & $5(41.7)$ & $5(33.3)$ \\
\hline Twice & $4(33.3)$ & $1(6.7)$ \\
\hline Three or more times & $1(8.3)$ & $0(0)$ \\
\hline \multicolumn{3}{|l|}{$\begin{array}{l}\text { Number of times missed ART } \\
\text { appointment }\end{array}$} \\
\hline Never & $2(16.7)$ & $10(66.7)$ \\
\hline Once & $541.7)$ & $3(20.0)$ \\
\hline Twice & $4(33.3)$ & $2(13.3)$ \\
\hline Three or more times & $1(8.3)$ & $0(0)$ \\
\hline \multicolumn{3}{|l|}{ Male partner age } \\
\hline $30-35$ & $3(25.0)$ & $3(20.0)$ \\
\hline $36-39$ & $2(16.7)$ & $7(46.7)$ \\
\hline 40 and older & $7(58.3)$ & $5(33.3)$ \\
\hline
\end{tabular}




$\begin{array}{lll}\text { Negative } & 3(25.0) & 8(53.3) \\ \text { Positive } & 9(75.0) & 7(46.7) \\ \text { Male partner accompanying to } & & \\ \text { ANC/ART clinic } & & \\ \text { Never } & 5(41.7) & 0(0) \\ \text { Once } & 6(50.0) & 4(26.7) \\ \text { Twice } & 1(8.3) & 6(40.0) \\ \text { Three or more times } & 0(0) & 5(33.3)\end{array}$
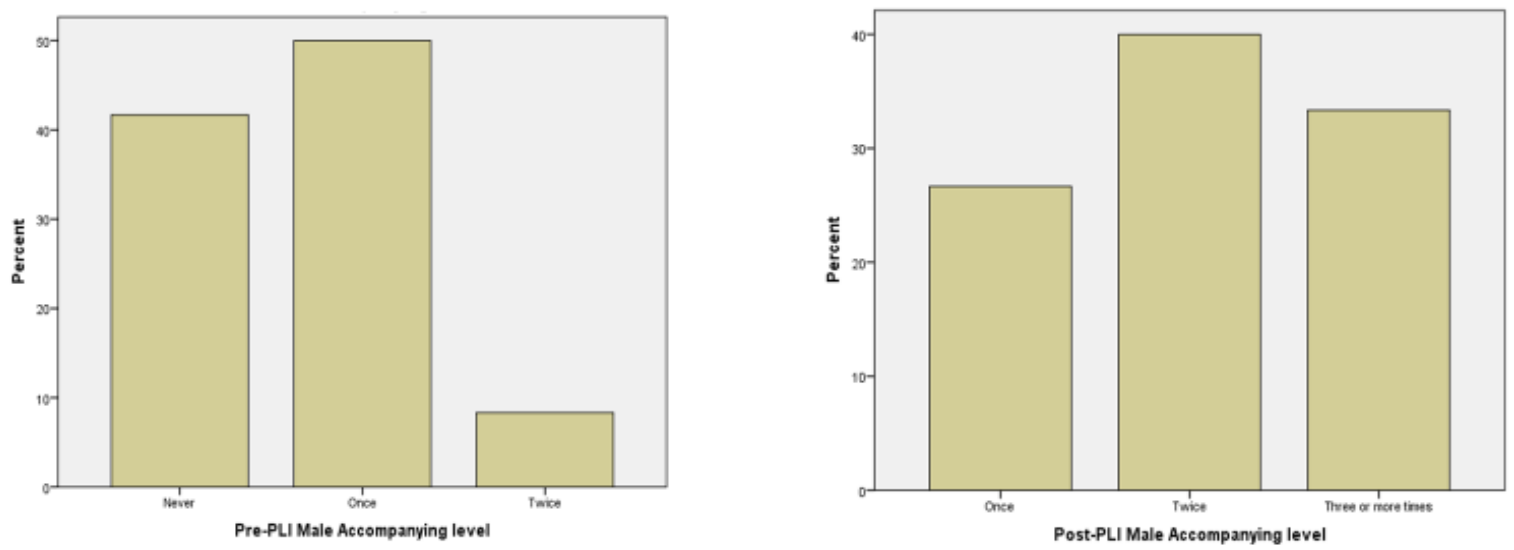

Figure 1: Number of times a male partner accompanied respondent to ANC/ART clinic

\section{ACKNOWLEDGMENT}

The authors thank the staff of secondary health facilities in Cross River State, their clients and the Field Assistants who helped in the data collection process.

\section{REFERENCES}

[1] A.A. Awofala, O.E. Ogundele, HIV Epidemiology in Nigeria, Saudi Journal of Biological Sciences, 25 (2018) $697-703$.

[2] UNICEF, Prevention of Maternal to Child Transmission (PMTCT), in, UNICEF, 2020.

[3] S. Theuring, L. Jefferys, P. Nchimbi, P. Mbezi, J. Sewangi, Increasing Partner Attendance in Antenatal Care and HIV Testing Services: Comparable Outcomes Using Written versus Verbal Invitations in an Urban Facility-Based Controlled Intervention Trial in Mbeya, Tanzania, PLoS One, 11 (2016) 1-13.

[4] UNICEF, NBS, 2016-2017 Survey Findings, in: N.B.o.S.a.U.N.C.s. Fund. (Ed.) Multiple Indicator Cluster Survey (MICS), UNICEF, Abuja, 2017.

[5] K. Hampanda, L. Abuogi, P. Musoke, M. Onono, A. Helova, E. Bukusi, J. Turan, Development of a Novel Scale to Measure Male Partner Involvement in the Prevention of Mother-to-Child Transmission of HIV in Kenya, AIDS Behav, 24 (2020) $291-303$.

[6] B.P. Giroir, The time is now to end the HIV epidemic, American Journal of Public Health, (2020) 22-24.

[7] N.F. Takah, I.T. Kennedy, C. Johnman, The impact of approaches in improving male partner involvement in the prevention of mother-to-child transmission of HIV on the uptake of maternal antiretroviral therapy among HIV-seropositive pregnant women in sub-Saharan Africa: a systematic review BMJ Open, 7 (2017) 1-11.

[8] A. Chadambuka, L. Leila Katirayi, A. Muchedzi, E. Tumbare, R. Musarandega, A.I. Mahomva, G. Woelk, Acceptability of lifelong treatment among HIV-positive pregnant and breastfeeding women (Option B+) in selected health facilities in Zimbabwe: a qualitative study, BMC Public Health, 18 (2018) 57.

[9] I. Triulzi, I. Palla, F. Ciccacci, S. Orlando, L. Palombi, G. Turchetti, The effectiveness of interventions to involve men living with HIV positive pregnant women in low-income countries: a systematic review of the literature, BMC Health Services Research, 19 (2019) 1-15.

[10] UNAIDS, HIV and AIDS in Nigeria, in, Joint United Nations Programme on HIV/AIDS, Geneva, Switzerland, 2019. 
[11] O. Omonaiye, S. Kusljic, P. Nicholson, E. Manias, Medication adherence in pregnant women with human immunodeficiency virus receiving antiretroviral therapy in sub-Saharan Africa: a systematic review, BMC Public Health, 18 (2018) 805.

[12] S.A. Spangler, M. Onono, E.A. Bukusi, C.R. Cohen, J.M. Turan, HIV-Positive Status Disclosure and Use of Essential PMTCT and Maternal Health Services in Rural Kenya, J Acquir Immune Defic Syndr, 67 (2014) S235-S242. 